Musées, Patrimoine et Culture scientifiques et techniques

$144 \mid 2012$

novembre - décembre 2012

\title{
Mieux vivre la nature à travers une visite au musée
}

\section{Anne Charpentier}

\section{OpenEdition \\ Journals}

Édition électronique

URL : http://journals.openedition.org/ocim/1139

DOI : $10.4000 /$ ocim. 1139

ISSN : 2108-646X

Éditeur

OCIM

Édition imprimée

Date de publication : 1 novembre 2012

Pagination : $33-41$

ISSN : 0994-1908

Référence électronique

Anne Charpentier, « Mieux vivre la nature à travers une visite au musée », La Lettre de l'OCIM [En ligne],

144 | 2012, mis en ligne le 01 novembre 2014, consulté le 01 mai 2019. URL : http://

journals.openedition.org/ocim/1139; DOI : 10.4000/ocim.1139 


\section{Mieux vivre la nature \\ à travers une visite au musée}

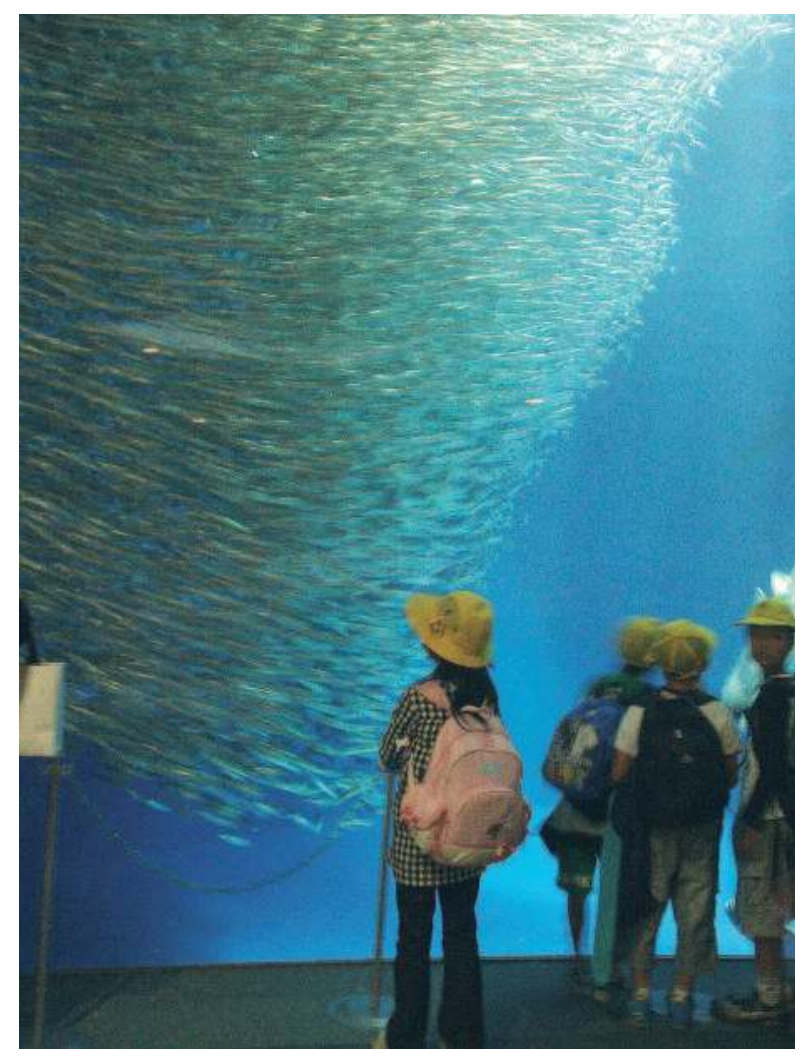

L'aquarium de Nagoya avec un effet immersif saisissant offert par un banc de sardines en mouvement. (C) Anne Charpentier

\footnotetext{
* Anne Charpentier est directrice de l'Insectarium de Montréal acharpentier@ville.montreal.qc.ca
}

\section{Anne Charpentier *}

Comment rendre les approches des musées de sciences naturelles encore plus efficaces pour relier les humains à la nature? Cette contribution propose quelques pistes en explorant des domaines et des approches qui pour la plupart se situent hors des sentiers habituels de la muséologie.

\section{L'urgence de " mieux vivre la nature »}

L'humain a graduellement acquis une certaine connaissance du vivant et de l'environnement, grâce aux observations, aux essais empiriques, à la domestication, aux explorations, aux Sciences de la Vie et de la Terre, ainsi qu'à l'effort de musées d'Histoire naturelle qui, à travers collectes, identifications et recherches, ont documenté et étudié la nature. Durant les quatre derniers siècles, les conceptions cartésiennes dichotomique et mécaniste de la nature, l'industrialisation, l'augmentation significative de la population mondiale, puis les comportements de surconsommation par une fraction de cette population, mettent à risque la diversité biologique de sorte que si rien ne change à court terme, c'est la survie de l'humanité sous cette forme connue, qui pourrait être menacée. Mieux vivre la nature devient une évidence, une pressante nécessité. Nous détenons toutes les clés pour établir une meilleure alliance avec la nature, et nos musées peuvent contribuer à en raviver l'usage.

\section{Quel rôle pour les musées de sciences naturelles?}

Outre leurs rôles toujours essentiels de conserver et de comprendre la nature, les musées de sciences naturelles 
ont aussi pour mission la diffusion de leurs savoirs et l'éducation à la nature. Ils le font à travers une panoplie d'outils pédagogiques et d'expositions ayant recours à divers modes de représentation du monde naturel. Ces modes de représentation ont suivi une évolution, notamment influencée par le développement des sciences de la vie et par les acteurs des institutions muséales. À l'origine, et c'est encore le cas pour certains musées, les expositions étaient conçues par les conservateurs des collections. Cela a donné lieu, par exemple, aux galeries d'anatomie comparée, de paléontologie ou à des salles consacrées à un groupe taxonomique : des approches centrées sur « la collection à montrer » et « la science à transmettre ». L'évolution des sciences de la nature vers des approches plus globales, telles que la biogéographie et l'écologie, a favorisé la mise en place des dioramas puis des expositions thématiques, où le thème définit les moyens muséographiques, le parcours et le choix des collections mises en valeur.

Depuis une vingtaine d'années, les musées se sont davantage questionnés sur leurs publics et se sont ouverts aux modes d'apprentissage, afin de rendre leurs expositions et programmes éducatifs plus accessibles et efficaces. Aujourd'hui, il est naturel que plusieurs intervenants contribuent à la réalisation des expositions et programmes : conservateurs et scientifiques bien sûr, mais aussi muséologues, éducateurs, designers, scénographes et artistes, pour ne citer que ces derniers.

Certains de ces modes de représentation thématiques offrent sans conteste une efficacité et une forme d'expérience appréciée par plus d'un. Le contact avec des objets et spécimens authentiques, mis en contexte, donne un sens à ce que l'on connaît déjà. Selon Falk et Dierking (2000) invariablement on y apprend quelque chose. Cela, nous le constatons et le mesurons dans nos musées. Toutefois, ces quelques informations « retenues » par nos visiteurs favorisent-elles une nouvelle relation à la nature ? À travers certaines approches, nous contribuons à changer les attitudes envers les insectes, par exemple. Cependant, arrivons-nous à induire un réel désir de changement profond et durable?

Les musées de sciences naturelles devraient tenter d'aller beaucoup plus loin en créant des expériences de visite et hors de leurs murs, qui contribuent à induire un tel changement. Il va de soi que l'enjeu de relier les humains à la nature dépasse largement la responsabilité de nos institutions. Cependant, qui de la société, sinon nos musées, est le mieux placé pour inspirer une telle évolution de paradigme?

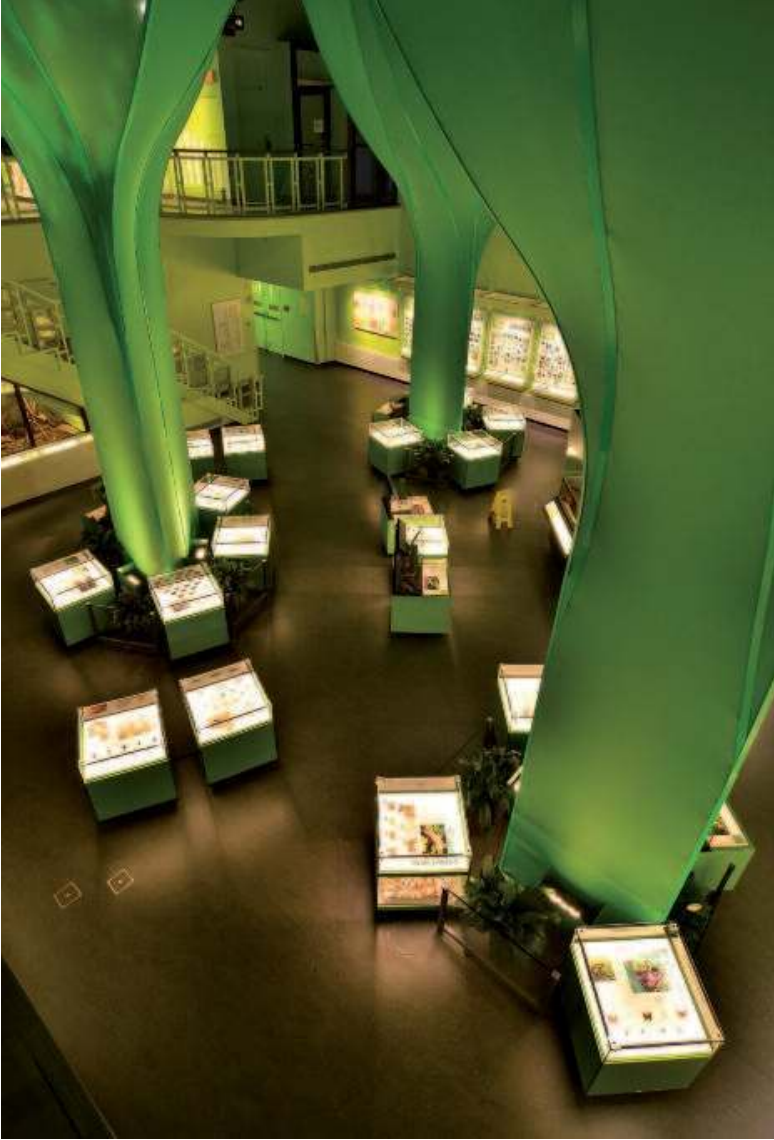

L'exposition Nous, les insectes ! à l'Insectarium de Montréal () Insectarium de Montréal

Comment nos musées peuvent-ils contribuer à relier l'humain à la nature ? Outre les études des publics de musées, les évaluations de l'efficacité des moyens muséographiques et les rudiments de pédagogie qui sont désormais connus des concepteurs, plusieurs domaines ont développé des savoirs fort complémentaires et parfois convergents, utiles à la présente réflexion. On pense aux études sociologiques sur la diversité des systèmes de valeurs par rapport à la nature ; aux avancées des connaissances en neurosciences qui nous permettent de mieux comprendre le fonctionnement émotif et cognitif des humains et les ajustements des approches pédagogiques basées sur ces connaissances ; à la psychologie du changement ; à la psychologie environnementale ; à l'écopsychologie ; à la psychologie de la conservation de la nature ; au courant de la «deep ecology » et aux réflexions développées autour de la notion de «biophilie».

L'exploration que nous proposons ici ne prétend pas construire une étude approfondie de chacun de ces domaines. Elle se veut une mise en lumière de connaissances, de pistes et d'outils, desquels les concepteurs d'expositions et de programmes pourraient s'inspirer pour faire en sorte que l'impact des expériences offertes à nos publics soit davantage significatif.

Ces explorations nous ont aussi amenés à mieux comprendre en quoi le contact avec la nature est un besoin fondamental au développement des humains et 
en conséquence, ont renforcé notre conviction de pousser davantage nos expériences muséales vers d'authentiques rencontres avec la nature.

\section{De l'éloignement de la nature}

Notre distanciation de la nature est le résultat de plusieurs causes et diverses interprétations coexistent à ce sujet. Certains affirment qu'elle remonte à notre besoin ancestral de sécurité, ayant favorisé, selon Roszak (1995, 2001), une séparation conceptuelle du dedans «in-here » et du dehors « out-there ». Les premiers humains organisaient leur survie autour de ressources et de cycles de la nature directement visibles autour d'eux, et notre cerveau a évolué en conséquence. Bien que les cycles globaux, comme celui des changements climatiques, nous soient révélés grâce aux technologies et aux modélisations, nous serions toujours placés devant notre limite évolutive à les comprendre, puisque leur échelle dépasse largement celle des cycles directement observables (Fisk, 2008).

Nos modes de vie se sont sédentarisés et urbanisés, de sorte qu'en 2006, $50 \%$ de la population mondiale vivait en milieu urbain (en 2010:85\% en France et $81 \%$ au Canada). Comme l'industrialisation, l'urbanisation et les technologies réduisent notre contact direct avec la nature, notre intérêt naturel pour la diversité des formes de vie serait redirigé vers des artéfacts fabriqués par les humains (Lemelin, 2011). Au Québec, les jeunes passent plus de 14 heures par semaine devant la télévision (Cardinal, 2010), sans compter celles passées sur Internet ou à écrire des

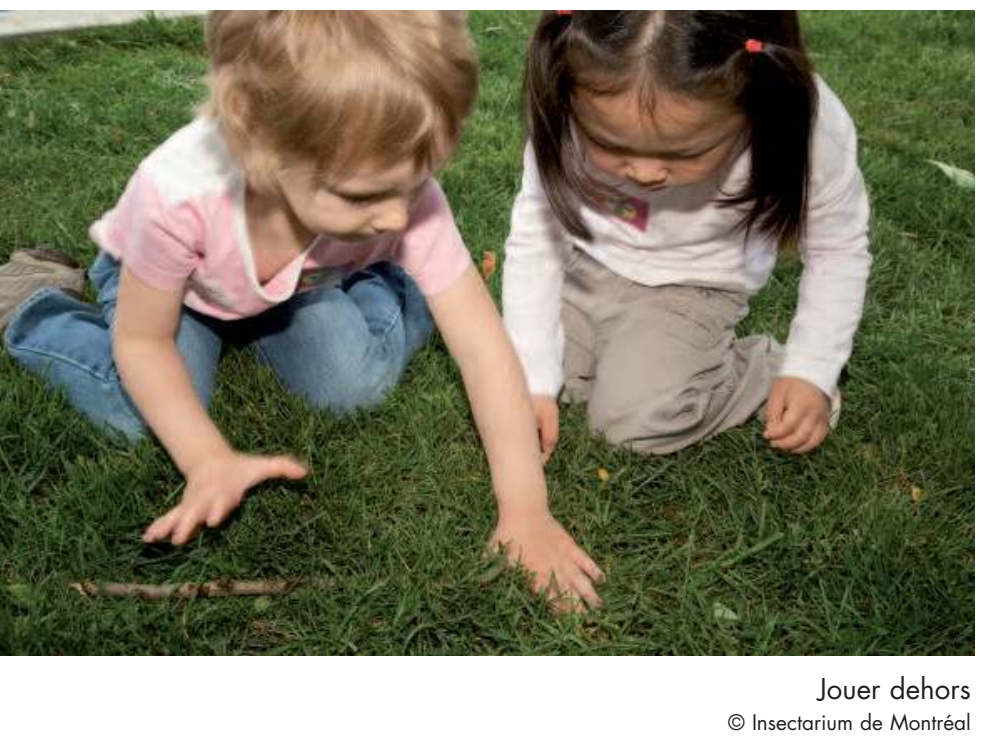

messages textes. Le développement de technologies, si utile soit-il, nous plonge à notre insu dans l'abstraction et incite à vivre par procuration dans des mondes imaginaires, coupés de nos sens. "For too long we've closed ourselves to the participatory life of our senses (...). We've taken our primary truths from technologies that hold the world at a distance » (Abram, 2010, pp. 6-7). À cet égard, l'ordinateur en réseau est un amplificateur nerveux particulièrement puissant. L'engourdissement qu'il produit l'est tout autant. L'ordinateur démultiplie les capacités de traitement de notre système nerveux et ce faisant, le modifie en diminuant sa capacité d'attention, de pensée et de créativité (Carr, 2012 ; Louv, 2005, 2011). Certains auteurs font état des mécanismes opérants de la télévision ou des jeux vidéo, et arguent que s'adonner à ces activités favorise le stress et une activation exogène des mécanismes de l'attention, réduisant la capacité de maintien autonome de cette capacité (Louv, 2011 ; Desmurget, 2012). On parle de vidéophilie (Pergams, 2007) et d'amnésie générationnelle de l'expérience directe avec la nature (Clayton et Myer, 2009). Les enfants risqueraient de devenir des adultes pour qui la nature n'est pas une priorité : voilà le public de nos musées.

\section{Les bienfaits du contact avec la nature}

Le manque de contact avec la nature autant que les bienfaits d'y être exposé sont bien documentés (Charles, Louv et Bodner, 2008 ; Cardinal, 2010 ; Frumkin, 2008 ; Louv, 2005 et 2011 ; Moore et Marcus 2008 ; Ulrich, 2008). Un manque de contact avec la nature aurait des effets néfastes sur le développement et la santé des humains, particulièrement des enfants. Privés de nature, ces derniers présentent moins d'autonomie créative pour des jeux non structurés et la propension augmente à développer de l'hyperactivité et un déficit d'attention, des désordres physiques (obésité, anorexie) et des désordres mentaux (dépression, agressivité, violence précoce, peurs).

Par ailleurs, être dans la nature, s'y exposer, favorise un développement sain, une meilleure santé mentale, émotionnelle et physique, diminue le stress, stimule la créativité et l'empathie. Plusieurs travaux de recherche relèvent une corrélation étroite entre la qualité de l'environnement, le bien-être, la santé et la capacité des humains à apprendre (Kellert, Heerwagen et Mador, 2008 ; Frumkin, 2008 ; Ulrich 2008, Clayton et Myer, 2009).

De plus, par sa variabilité et son imprévisibilité, la nature offre de multiples situations de jeu qui favorisent à leur tour, chez les enfants, une perception 
positive et le développement d'un sens de connexion avec elle. Etre en contact avec la nature et jouer en plein air, puis apprendre à la respecter en compagnie d'un mentor, favorisent le développement d'une meilleure conscience environnementale chez les jeunes (Charles, Louv, Bodner et Guns, 2008 ; Lester et Maudsley, 2006 ; Clayton et Myer, 2009).

Plusieurs auteurs appuient l'hypothèse que les humains auraient une prédisposition naturelle à aimer et à interagir avec la nature. Cette hypothèse, nommée «biophilie » (Wilson, 1984), donne lieu au développement de nouvelles recherches sur la santé et le bienêtre qui n’ont pas cessées de confirmer la nécessité de relier l'humain à la nature. Nous y revenons plus loin. La nature nous prédispose à mieux apprendre et à mieux être. Pourquoi donc en est-il ainsi ? La nature nous sollicite de façon globale à travers nos cinq sens, elle fait émerger des émotions, elle offre inspiration, étonnement, contrastes et variété. Elle favorise également des jeux plus créatifs chez les enfants et aide à se libérer d'une fatigue due à une attention trop unidirectionnelle. Par le toucher, elle sollicite notre processus de reconnaissance, contribue à l'apaisement et au sentiment d'empathie. Nous avons évolué grâce à la nature. Ces attributs, nous le verrons, présentent des concordances avec nos connaissances récentes sur le fonctionnement du cerveau humain et nos modes d'apprentissage.

\section{Apprendre la nature}

Les réactions d'insécurité et même de crainte à l'égard de certains éléments naturels sont communes.

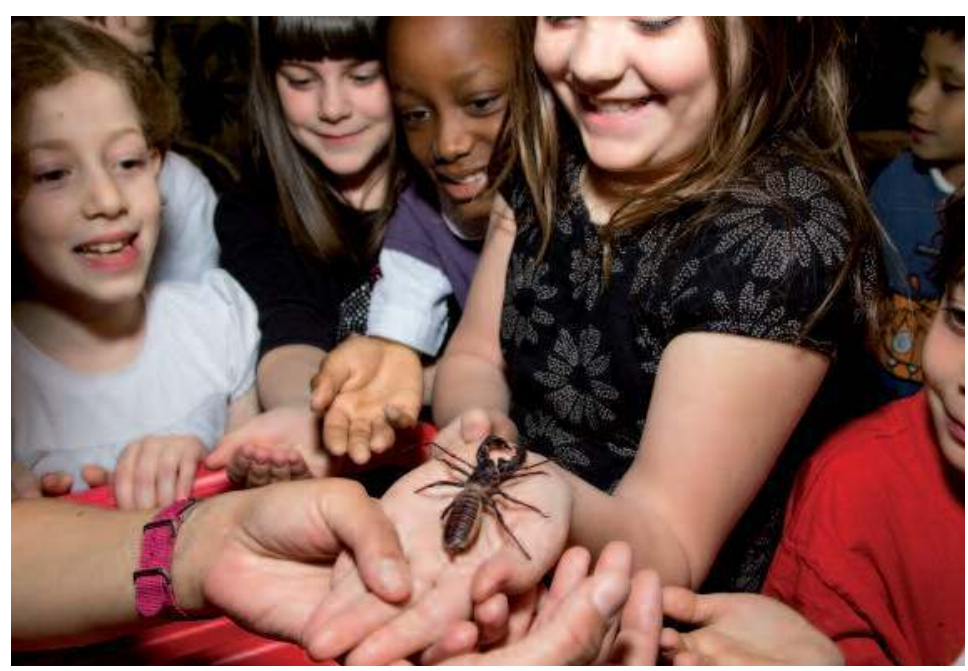

Manipuler les insectes pour apprendre à les connaître. (c) Claude Lafond
À l'Insectarium de Montréal, un espace pour la vie, nous observons chez des visiteurs une méfiance ou un dédain face aux insectes. Dans le contexte actuel où notre distanciation avec la nature est notable, il nous apparaît souhaitable de contrer d'abord ce que Sobel (1996) a nommé « l'écophobie », à savoir l'anxiété causée lorsqu'on est confronté trop jeune à des catastrophes naturelles ou la simple peur de la nature due à sa non familiarité.

Les neurosciences révèlent depuis quelques années le fonctionnement fabuleux de notre cerveau et fournissent de plus en plus d'informations applicables à la psychologie et à la pédagogie. À titre d'exemple, réduire l'apparence de menace diminuerait le stress inconscient, qui freine l'ouverture au nouveau et à l'apprentissage. De plus, pour favoriser l'apprentissage, mieux vaut savoir que les formulations négatives du langage sont difficilement assimilables par le cerveau et que d'insister sur des problèmes revient à les ancrer et à masquer les solutions (Jensen, 2005 ; Kourilski, 2008 ; Clayton et Myer, 2009). Ainsi, afin de mieux engager nos visiteurs, il serait opportun de miser sur les leviers suivants :

- installer un climat positif pour contrer « l'écophobie »; - avoir recours à des émotions positives universelles ;

- faire des liens pertinents avec les références de nos visiteurs ;

- leur offrir des choix d'expériences à vivre ;

- capter l'attention par de la nouveauté, des événements ou faits étonnants, des contrastes, de la variété (attributs largement présents dans la nature) ; - faire en sorte que nos animateurs/médiateurs communiquent leur passion et leur enthousiasme ;

- engager la globalité de la personne par le mouvement corporel, la célébration, le rituel, la possibilité de s'exprimer et la rétroaction, le partage d'histoire de vie, le faire soi-même/ensemble et la coopération.

Le domaine de la psychologie du changement présente également des pistes afin d'élaborer des programmes éducatifs et des expositions encore plus efficaces pour induire des changements d'attitudes et de comportements chez nos publics :

- faire référence à des valeurs présentes chez le public cible (CSoP Reasearch \& Consulting, 2009);

- faire référence à une norme sociale : « tout le monde le fait... fais le donc! » (Manfredo, 2008) ;

- avoir recours à la métaphore pour rendre accessibles des concepts compliqués ;

- proposer visuellement des solutions et des gestes inspirants, explicites, accessibles et observables ;

- utiliser un ton raisonnable et non alarmiste, car ce dernier rend le public sceptique ; 


\section{Monarque sans frontière : le pouvoir de changer les choses}

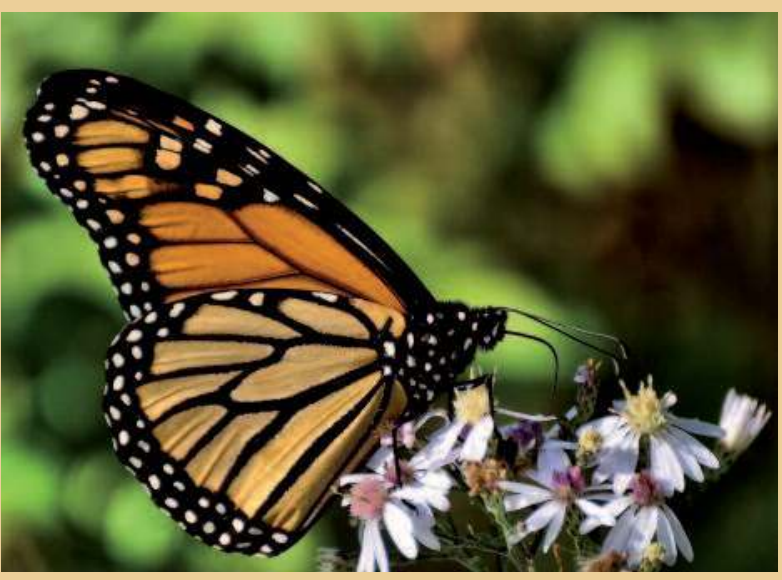

Le Papillon Monarque (c) André Sarrazin

Au-delà de la visite du musée, certains programmes de sciences participatives comportent plusieurs approches très efficaces pour relier l'humain à la nature et induire des changements d'attitudes et de comportements. Le programme Monarque sans frontière de l'Insectarium, en est un bel exemple.

\section{Contribuer à la science \\ et prendre soin de la nature}

Imaginez un instant que des scientifiques souhaitent documenter l'habitat d'un papillon menacé et que cet habitat s'étende sur plus de 100 millions d'hectares... La population de monarques d'Amérique, dont font partie ceux du Québec, est la plus impressionnante, tant par sa taille (plus de $80 \%$ des monarques du monde) que par l'ampleur de sa migration nord-sud (près de $4000 \mathrm{~km}$ ). Devant l'urgence de préserver la migration du monarque, des scientifiques de l'université du Kansas ont mis en place Monarch Watch, un programme qui implique des citoyens à l'échelle de ce vaste territoire.

Monarque sans frontière, développé par l'Insectarium, un espace pour la vie, contribue à cet effort de recherche et de conservation. Chaque année, plus de 1000 trousses d'élevage comportant des chenilles, des chrysalides et une asclépiade, sont distribuées à des groupes scolaires et des citoyens. Durant environ 3 semaines, ces participants consignent chaque jour leurs observations des chenilles et de la métamorphose des chrysalides en papillons, puis les libèrent à la fin de l'expérience, dûment étiquetés. Ils découvrent ainsi les multiples facettes de la vie des monarques et s'émerveillent à coup sûr devant la beauté de chacune des étapes de leur vie.
Après cette expérience, impossible de ne pas ressentir un attachement bien particulier pour ce magnifique papillon orangé et souhaiter agir pour le protéger. L'émerveillement, la contribution à la science et le fait d'agir envers la nature, sont autant de facteurs qui favorisent une grande satisfaction et... le bien-être que procurent aux participants les gestes simples de prendre soin d'un papillon et d'une plante. Pour les participants à ce type de programme, l'investissement en temps est minime, comparé à ce qu'il procure comme expérience de contact direct et mémorable avec la nature. L'impact de tels programmes sur les attitudes et les comportements des participants est hautement significatif, d'autant qu'ils représentent désormais des avenues incontournables pour les scientifiques, auxquels ils offrent de précieuses données.

\section{Le futur est... monarque !}

Dès le printemps 2013, l'Insectarium poursuit sa volonté d'impliquer encore davantage les citoyens en les invitant à créer chez eux des oasis pour les monarques, qui offriront à ces papillons leurs plantes nourricières favorites et celles spécifiques à leur reproduction. Guide pratique et programme de reconnaissance, sous forme d'accréditation, sont quelques-uns des outils prévus pour inciter les citoyens à prendre part à cette initiative, qui s'inscrit dans le mouvement de l'Espace pour la vie pour mieux vivre la nature.

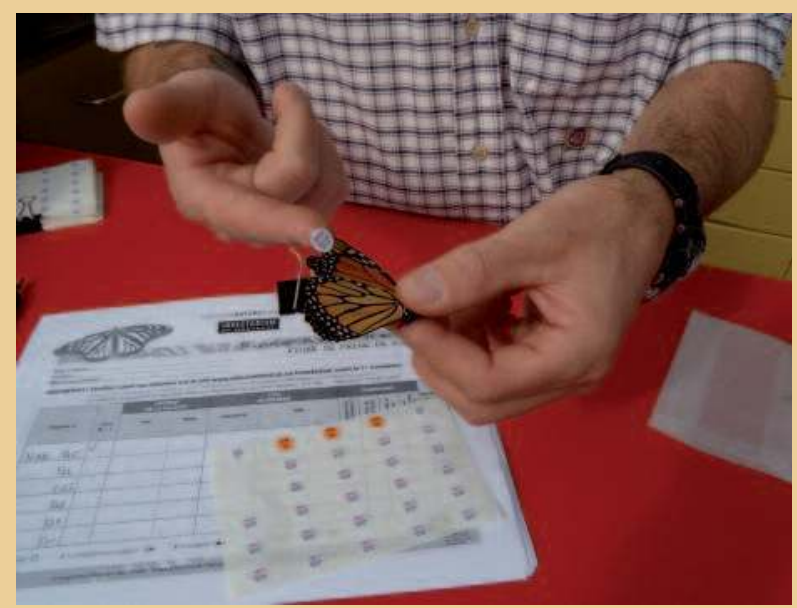

La fiche de données concernant le Monarque ๑) André Payette 
- la crédibilité du messager est déterminante ;

- donner des idées d'actions est plus efficace que de mettre l'emphase sur les enjeux environnementaux ; - engager les publics à travers les programmes de sciences participatives ;

- rapporter quelque chose chez soi, qui rappelle le geste à poser et s'insère dans le quotidien ;

- stimuler la confiance envers le futur ;

- faire appel à la quête de sens des personnes.

\section{Re-sentir la nature}

Les approches abordées précédemment visent déjà des apprentissages et des changements de comportement face à la nature. Sans sous estimer l'importance de ces dernières, comment nos musées peuvent-ils dépasser la représentation de la nature, dépasser le cadre classique d'une visite pour faire vivre une expérience de présence à la nature ? Bien que les thèmes et les contenus scientifiques demeurent nécessaires, comment informer sur la nature autrement que par des mots, des images et des médiations ? Est-ce là une quête utopique ?

Nos sens et notre corporalité «toute animale » demeurent nos premières sources d'information, bien avant l'intellect. Pourquoi s'en priver? Selon Laura Sewall (1995), une psychologue de la perception, l'atrophie de nos sens serait au cœur de la crise environnementale actuelle. Les raviver pourrait être un pas important pour nous relier à nouveau à la nature.

Les expériences offertes par nos musées auraient intérêt à revisiter et solliciter davantage tous les sens des visiteurs, de façon beaucoup plus affirmée. Une expérience forte de rencontre avec la nature permet une perméabilité et une fluidité entre le soi et nos apparentes frontières avec la nature. David Abram (2010) insiste sur l'importance de nous reconnecter à notre animalité à travers tous nos sens. Nos activités mentales dépendent autant de notre cerveau que de l'expérience de tout notre corps avec l'environnement immédiat. Qui n'a pas déjà noté l'effet d'un paysage ou d'un changement d'ensoleillement sur ses états d'âmes ?

Sewall (1995) offre cinq «pratiques perceptuelles » qui s'appuient principalement sur le fonctionnement du sens de la vision et qui nous permettraient de mieux percevoir notre « condition écologique » :

- La première vise à réapprendre à être pleinement conscient, à prêter attention, à se concentrer, à prendre le temps et le soin d'observer les textures, les formes, les couleurs... puis à faire appel à notre capacité naturelle

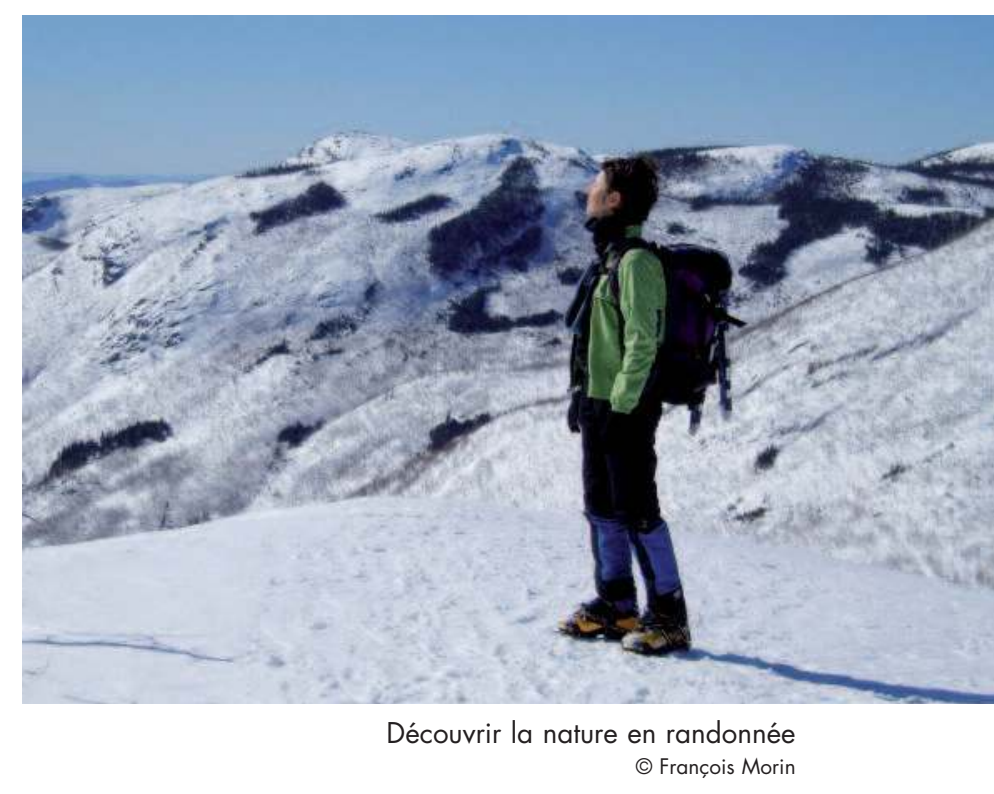

à repérer la nouveauté. Cette capacité de repérer la nouveauté dans la nature nous a permis d'évoluer et de survivre.

- La seconde, fait référence à notre propension à percevoir les objets et les éléments de la nature comme indépendants les uns des autres et conséquemment à notre difficulté à décoder des relations, dont la nôtre à la biosphère. Un défi pour nos musées est de mettre en évidence ces relations à travers les iconographies et les histoires que nous racontons. Ces deux premières «pratiques » demandent du temps et dans un contexte de visite muséale, il importe de concevoir des expériences où l'on arrive à recréer des espaces/temps signifiants.

- La troisième consiste à faire appel à notre capacité à identifier des patrons familiers à travers le chaos ou la variabilité de la nature. Les voies à explorer, tant pour leur impact sur notre perception de l'espace que du temps, seraient les illusions visuelles qui jouent avec la profondeur, la perspective, le contour (par exemple, celui d'un animal parfaitement camouflé sur une surface de même texture) et le recours aux fractals (que nous décodons aisément).

- La quatrième repose sur notre capacité à percevoir la proximité et la profondeur, à savoir la perspective. La perspective engage notre corps tout entier. Elle nous permet de nous situer physiquement dans le paysage et dans l'espace (Abram, 2010). Sentir la perspective, par exemple du fond d'un canyon ou du haut d'une montagne, nous place en position de vulnérabilité et favorise le sens d'être à même la nature, plutôt qu'à côté d'elle. 
- La cinquième « pratique » souligne l'importance de nourrir notre capacité d'imagination. Les images que nous créons et portons en nous, influencent en retour notre perception de la réalité, nos choix et conséquemment notre futur. Abram arguë que même les mots que nous utilisons pour exprimer notre relation à la nature ont à leur tour un profond effet sur ce que l'on voit, entend et apprécie du monde. Décrire la nature de façon mécaniste comme des séries d'objets inertes nous éloignerait de nos expériences sensorielles. Prendre soin de notre capacité à imaginer par soi-même, puis porter attention à notre façon de raconter la nature, deviennent une prescription au rétablissement de nos liens avec la nature. Qui pourrait croire que notre imagination puisse être uniquement stimulée par un monde construit d'aménagements et d'objets de fabrication humaine, sans les sensations que nous procure le contact avec la nature ? L'exploration du sens de la vision que nous venons d'aborder, pourrait être faite pour l'ensemble des sens.

\section{La nature intégrée au design}

La «biophilie » est une hypothèse avancée d'abord par le biologiste Edward O. Wilson (Wilson, 1984), qui stipule que les humains ont une affinité innée avec la nature issue de leur évolution biologique, qui se traduit par une recherche subconsciente de se connecter avec le vivant. Puis Kellert (Kellert et Wilson, 1993 ; Kellert, 2005) la définit comme un héritage génétique qui nous incite à valoriser la nature pour les bienfaits et le bien-être qu'elle nous procure. Puisqu'elle est si profondément ancrée dans notre biologie et notre évolution, la «biophilie» devient aussi un argument important pour protéger la nature.

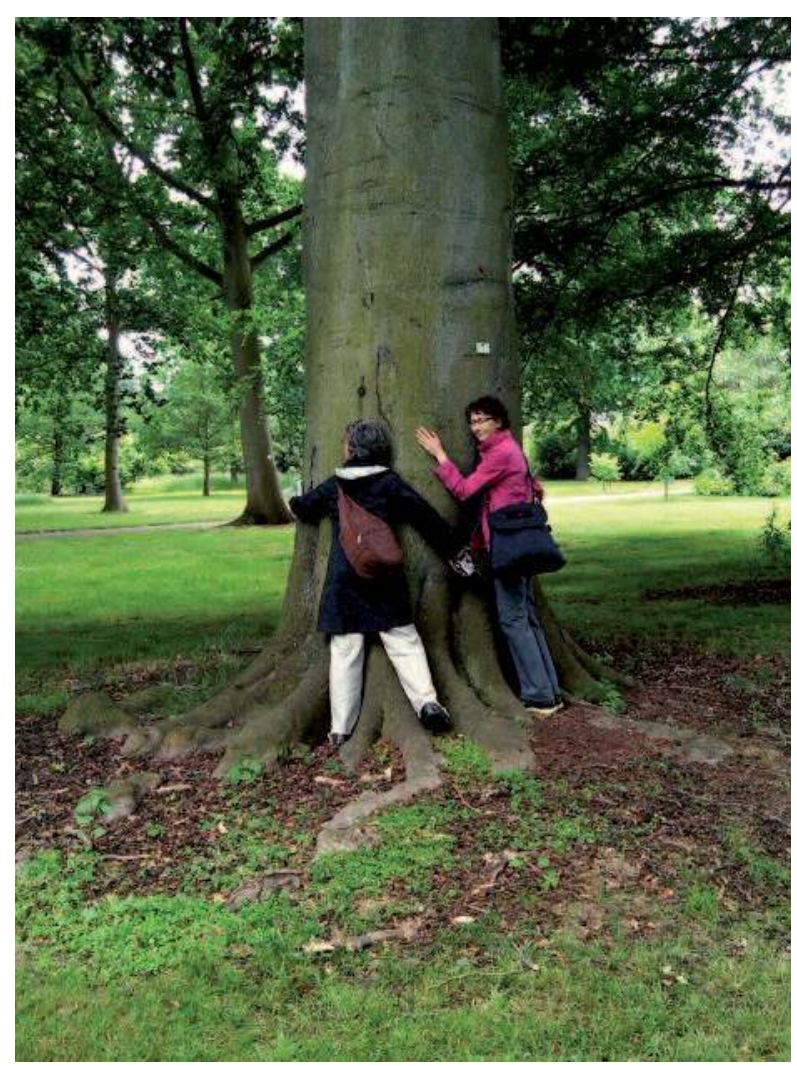

Accolade avec un arbre : sentir la nature (C) Rachel léger

Kellert et plusieurs collaborateurs (Kellert, Heerwagen et Mador, 2008) ont exploré les applications de ce concept au design d'intérieur, à l'architecture et à l'aménagement du paysage. Ils avancent que si les aménagements et les constructions que nous concevons tenaient davantage compte de nos besoins de nature, la distanciation que nous vivons avec elle serait moindre. Dans ce récent ouvrage collectif, les principes de design « biophilique » et les bénéfices de cette approche sont explicités. En voici quelques-uns : - avoir recours aux caractéristiques et éléments de la nature : eau, lumière naturelle, couleurs, plantes et

\section{"Living lab » Métamorphose}

Certaines idées avancées dans ce texte ont été mises en avant par l'Espace pour la vie (Biodôme, Insectarium, Jardin botanique et Planétarium de Montréal) en mai 2012, dans le cadre d'un exercice collectif de visualisation et de co-création (« Living lab », www.living-lab.ca) consacré au projet d'agrandissement de l'Insectarium de Montréal.

Une trentaine de personnalités et d'experts internationaux des domaines de l'entomologie, des sciences naturelles, de la muséologie, du design d'expériences immersives, du design « biophilique », de l'architecture verte, des artistes et des philosophes, ont été réunis avec autant d'experts de l'Espace pour la vie afin d'imaginer les orientations de ce projet, qui vise à susciter des rencontres authentiques avec les insectes et à reconnecter l'humain à la nature.

Avec ce premier «Living lab " l'Espace pour la vie explorait ainsi une approche de conception intégrée, où les participants étaient invités à dépasser leur cadre en répondant à la question "Qu'est-ce qui n'a pas encore été fait ? ", et à partager leurs idées sur le monde des insectes, sur les expériences immersives participatives ainsi que sur le design. 
murs végétalisés, animaux, matériaux naturels, vues sur la nature...;

- représenter et évoquer la nature par des motifs, textures et formes de la nature ;

- intégrer des propriétés, des processus et des cycles naturels au bâti ;

- jouer avec les diverses qualités de lumière naturelle et d'espace (dedans/dehors) ;

- tenir compte de la géographie, de la culture et de l'histoire locale ;

- tenir compte de nos besoins ancestraux : beauté, protection, sécurité...

Ces principes peuvent et devraient être davantage intégrés aux processus de conception de programmes et d'expériences muséales, si tant est que ces derniers visent à relier nos visiteurs avec la nature. D'autres approches en design et en architecture sont complémentaires au design « biophilique ». Le « Living Building Challenge » fait appel à une vision intégrée et holistique des fonctions naturelles dans les aménagements et le bâti. Imaginez un musée de la nature dont le fonctionnement même s'inspirerait de cette dernière.

\section{L'humain et la nature au cœur de nos processus imaginatifs}

En terminant, comment rendre les approches de nos musées encore plus efficaces pour relier les humains à la nature ?

À l'issue de ce bref survol, nous proposons une approche de conception beaucoup plus globale et multidisciplinaire, où les diverses expertises seraient mises en présence pour co-créer de façon intégrée. Il serait également souhaitable que les concepteurs s'intéressent d'abord à l'expérience à faire vivre aux visiteurs avant d'en imaginer le contenant, l'écrin. Les concepteurs pourraient davantage tenir compte des processus qui favorisent l'apprentissage et le changement chez les personnes, puis des systèmes de valeurs et du contexte social des visiteurs. Finalement, les informations scientifiques devraient être intégrées de façon plus « organique » à une expérience de rencontre avec la nature, afin que cette dernière fasse sens. Faire vivre notre discours pour faire sens contribuerait à propulser nos musées à l'avant-garde des rendez-vous avec la nature : n'est-ce pas là notre mission ?

\section{Bibliographie}

Abram, D. Becoming Animal, An Earthly Cosmology. New York : Pantheon Books, 2010, 313 p
Cardinal, F. Perdus sans la nature, Montréal : Québec Amérique, 2010, $201 \mathrm{p}$.

Carr, N. À propos des risques de laisser la frénésie s'emparer de notre âme, Nonveau Projet 01 : (Sur) vivre au 21e siècle. Montréal, printemps 2012, pp. 74-79.

Charles, C., Louv, R., Bodner, L., et Guns B. A Report on the Mouvement to Reconnect Children to the Natural World. Children \& Nature Network, 2008, 43 p.

Clayton, S. et Myers, G. Conservation Psychology : Understanding and promoting human care for nature. Oxford : Wiley-Blackwell, 2009, 253 p.

CSoP Research \& Consulting. How Canadians Value Nature : A Strategic and Conceptual Review of Literature and Research. Étude réalisée pour le compte d'Environnement Canada, 2009, 174 p.

Desmurget, M. Médias modernes et passivité attentionnelle, Cerveau et psycho, $\mathrm{n}^{\circ} 47,2012$, pp. 54-57.

Falk, J.-H. et Dierking, L.-D. Learning from Museums, Visitor Experiences and the Making of Meaning. Walnut Creek : Altamira Press, 2000, 272 p.

Fisk III, P. The Greening of the Brain, in Kellert, S.-R., Heerwagen, J.-H. et Mador, M.-L. Biophilic Design: The Theory, Science, and Practic of Briging Buildings to Life. Hobokem : John Wiley \& Sons, Inc, 2008, pp. 307-312.

Frumkin, H. Nature Contact and Human Health : Building the Evidence Base. in Kellert, S. R., Heerwagen, J.-H. et Mador, M.-L. Biophilic Design: The Theory, Science, and Practic of Briging Buildings to Life. Hobokem : John Wiley \& Sons, Inc, 2008, pp. 87-106.

Jensen, E. Teaching with the brain in mind. Alexandria : ASCD, 2005, 2e édition, $187 \mathrm{p}$.

Kellert, E.-O. et Wilson, E.-O. The Biophilia hypothesis. Washington : Island Press, 1993.

Kellert, S. Building for Life: Designing and Understanding the HumanNature Connection. Washington DC : Island Press, 2005, 250 p.

Kellert, S.-R., Heerwagen, J.-H. et Mador, M.-L. Biophilic Design, The Theory, Science, and Practic of Briging Buildings to Life. Hobokem : John Wiley \& Sons, Inc, 2008, 385 p.

Kourilsky, F. Du désir au plaisir de changer. Paris : Dunod, 2008, 4e édition, $340 \mathrm{p}$.

Lemelin, R.-H. To bee or not to bee : whether 'tis nobler to revere or to revile those six-legged creatures during one's leisure. Leisure Studies, 2011. 
Lester, S. et Maudsley, M. Play naturally, A review of Children's Natural Play. Commissioned for Playday, Londres : Playday, 2006, 105 p.

Louv, R. Last Child in the Woods : Saving Our Children from NatureDeficit Disorder. Chapel Hill : Algonquin Books, 2005, 334 p.

Louv, R. The Nature Principle. Chapel Hill : Algonquin Books, 2011. $317 \mathrm{p}$.

Manfredo, M.-J. Who Cares About Wildlife, Social Science Concepts for Exploring Human-Wildlife Relationships and Conservation Issues. New York : Springer, 2008, 228 p.

Moore, R.-C. et Cooper Marcus, C. Healthy Planet, Healthy Children : Designing Nature into the Daily Spaces of Childhood, in Kellert, S.-R., Heerwagen, J.-H. et Mador, M.-L. Biophilic Design: The Theory, Science, and Practic of Briging Buildings to Life. Hobokem : John Wiley \& Sons, Inc, 2008, pp. 153-203.

Pergam, O.-R.-W. et Zaradic, P.-A. Videophilia : Implications for Childhood Development and Conservation, The Journal of Developmental Processes, vol. 2, n 1,2007 . pp. 130-144

Roszak, T., Gomes, M.-E. et Kanner, A.-D. Ecopsychology : Restoring the Earth/Healing the Mind. Sierra Club Books, 1995.

Roszak, T. The voice of the Earth, An Exploration of Ecopsychology. Grand Rapids : Phanes Press, in An Alexandria Book, 2001, 2e édition.
Sewall, L. The Skill of Ecological Perception, in Roszak, T., Gomes, M.E. et Kanner, A.-D. Ecopsychology : Restoring the Earth/ Healing the Mind. Sierra Club Books, 1995.

Sobel, D. Beyond Ecophobia, Reclaiming the Heart in Nature Education. Great Barrington : The Orion Society, Nature Literacy Series, 1996, 45 p.

Ulrich, R.-S. Biophilic Theory and Research for Healthcare Design. in Kellert, S.-R., Heerwagen, J.-H. et Mador, M.-L. Biophilic Design : The Theory, Science, and Practice of Briging Buildings to Life. Hobokem : John Wiley \& Sons, Inc, 2008, pp. 87-106.

Wilson, E.-O. Biophilia : The Human Bond with Other Species. Cambridge : Harvard University Press, 1984.

\section{Sites Internet}

Satistiques sur les populations urbaines mondiales : www.statistiques-mondiales.com/population_urbaine.htm

Design « biophilique » : www.biophilicdesign.net/

Living Building Challenge : https://ilbi.org/lbc 